\title{
Geschichte ,von unten' mit dem Bilderbuch Die Lisa. Didaktisches Konzept, Möglichkeiten, Beispiele
}

Edith Wegerle-Johansen

Gjøvik Videregående Skole

\section{Zusammenfassung}

Der Artikel präsentiert einen theoretischen Rahmen, ein didaktisches Konzept und Aufgabenbeispiele für die Auseinandersetzung mit der deutschen Geschichte anhand des Bilderbuchs Die Lisa von Klaus Kordon und Peter Schimmel (1999). Der theoretische Rahmen erläutert $u$. a., dass die Bildbetrachtung mit einer gewissen Verlangsamung des Betrachtungsvorgangs einhergehen muss, um Details auf den Bildern entdecken zu können, die man durch eine kreativ-produktive Arbeitsweise weiter entfalten und vertiefen kann. Diese Arbeitsweise ist im fremdsprachlichen Deutschunterricht, wo Sprachbarrieren bedeutsam sind, für die Lerner besonders reizvoll.

Die Ergebnisse einer Fallstudie, die im Hochschul- und Sekundarbereich in Norwegen durchgeführt wurde, zeigen, dass die Lernenden ein tieferes Verständnis für die geschichtlichen Ereignisse in Deutschland entwickelt haben und dass die Kompetenzziele Verständnis und Toleranz, die im norwegischen Lehrplan LK-06 für den fremdsprachlichen Deutschunterricht hervorgehoben werden, erreicht werden können. Während des Arbeitsprozesses bekommen die Lernenden die Möglichkeit, sich auf die Figuren im Text näher einzulassen und sich mit deren Handlungsmotiven auseinanderzusetzen. Dadurch können sie zu Einsichten in Bezug auf kulturelles Verständnis und Toleranz gelangen, die auch einen gewissen Übertragungswert über das Buch hinaus besitzen.

Schlüsselwörter: Bilderbuch, Geschichte ,von unten', didaktisches Konzept, Deutsch als Fremdsprache

\section{Einleitung}

Literarische Texte spielen im fremdsprachlichen Deutschunterricht in Norwegen meist eine untergeordnete Rolle. Lesen als Informationsentnahme von eigens für den Unterricht verfassten Texten steht im Vordergrund, wodurch das Besondere, was das Lesen von literarischen Texten ausmacht, nur unzureichend beschrieben und ergründet werden kann. Die hier vorgestellte Untersuchung möchte einen empirischen Beitrag dazu leisten, die ästhetischen Möglichkeiten, die in einem relativ einfachen literarischen Text stecken und für die Auseinandersetzung mit Literatur zentral sind, im Unterricht nutzbar zu machen. Am Beispiel des Bilderbuchs Die Lisa. Eine deutsche Geschichte (1999) von Klaus Kordon (Text) und Peter Schimmel (Illustrationen) soll dargestellt werden, wie sowohl Studenten während des Grundfachstudiums Deutschsprachige Literatur und Kultur an einer norwegischen Universität als auch Lerner an der gymnasialen Oberstufe (Sekundarstufe II) mit dem Buch 
und dessen ästhetischen Möglichkeiten gearbeitet haben. Die Arbeit mit dem Bilderbuch Die Lisa im fremdsprachlichen Deutschunterricht soll mit dem nachfolgenden Zitat aus dem norwegischen Lehrplan LK-06 legitimiert werden:

Arbeid med ulike typer tekster og møte med kulturelle uttrykksformer fra målspråklandet kan utvikle interesse, forståelse og toleranse og fremme innsikt i ens egne livsvilkår og identitet. Det kan også bidra til leseglede, opplevelse og personlig utvikling. (Utdanningsdirektoratet, 2006)

Im Fremdsprachenunterricht soll demnach u. a. mit verschiedenen Textsorten gearbeitet werden, damit die Lernenden Interesse, Verständnis und Toleranz entwickeln können, wobei sie Einsichten in ihre eigenen Lebensbedingungen und in ihre Identität gewinnen sollen, was zu Lesefreude, Erlebnissen und persönlicher Entwicklung beitragen kann.

Die übergeordnete Fragestellung dieser Untersuchung ist, wie die Auseinandersetzung mit der Lebensgeschichte der Hauptfigur zur Vertiefung der Geschichtskenntnisse im Deutschunterricht und zur Entwicklung von Verständnis und Toleranz beitragen kann. In beiden Lernergruppen sollte das ästhetische Potential des Bilderbuchs durch eine ,kreativproduktive" Arbeitsweise nutzbar gemacht werden. Ziel dabei war, dass sich die Lernenden anhand der Lebensgeschichte einer relativ, einfachen' Frau aus ihrer eigenen Perspektive mit der deutschen Geschichte von der Kaiserzeit bis zur Nachwende (ca. 1899 bis in die 1990er Jahre) auseinandersetzen. Zur Bearbeitung der Aufgaben wurden u. a. mediale Hilfsmittel genutzt, wobei wichtig war, dass die Lernenden ihre Deutungen wieder mit der Protagonistin bzw. den anderen Figuren im Buch vernetzen.

Dadurch, dass die deutsche Geschichte hauptsächlich durch das, was der Hauptfigur widerfährt, vermittelt wird, erhalten die Lernenden die Möglichkeit, das Zusammenspiel von Kognition, Emotion und Ethik an einem literarischen Text zu erfahren. Neben kognitiv orientierten Texterschließungsverfahren sollen kreative und produktive Handlungsformen zur Anwendung kommen, wobei emotionale Prozesse bei den Lernern in Gang gesetzt werden, die zur Entwicklung von Verständnis und Toleranz beitragen können.

Bevor auf den Toleranzbegriff im Lehrplan LK-06, auf den Begriff Geschichte ,von unten“ und die Besonderheiten der Gattung Bilderbuch eingegangen wird, seien einige kurze Überlegungen zur Verwendung eines Kinderbuchs im Hochschul- und Sekundarbereich anzustellen. Im Hochschulbereich wurde das Bilderbuch am Ende des zweiten Semesters im Studium Auslandsgermanistik eingesetzt, um den geschichtlichen Kontext der Pflichtlektüre noch einmal zusammenzufassen. In der Sekundarstufe wurden Teile des Lehrbuchs, die die deutsche Geschichte faktenreich darstellen, durch die Arbeit mit dem Bilderbuch ersetzt. Dadurch sollte der Schwerpunkt von der Informationsentnahme auf die genannten Lernziele gelenkt werden. Anhand der Präsentation der kreativ-produktiven Arbeiten, den „dichten“ Beschreibungen (Geertz 1987, 7-43), während des Arbeitsprozesses und anhand von Antworten auf einen Fragebogen, den die Lernenden im Anschluss an die Arbeit ausgefüllt haben, werden wesentliche Funde vorgestellt. Der Fragebogen wird ergänzend herangezogen, hier sollten die Lerner noch einmal darüber reflektieren, ob sie sich mit dem Thema Toleranz auseinandergesetzt haben. Aus dem vorgestellten Material ergibt sich, dass der Prozess den eigentlichen Inhalt der Arbeitsweise ausmacht. 


\section{Empathie, Verständnis und Toleranz}

Dadurch, dass sich die Lernenden intensiv mit Text und Bildern auseinandersetzen und den von ihnen gewählten Zeitabschnitt aus Lisas Leben mit ihrem Hintergrundwissen bzw. ihrem intertextuellen Wissen bereichern, dringen sie tiefer in das Leben der Hauptfigur ein. Lesen wird somit zu einem Prozess, der sich von der bloßen Faktenaneignung beim Lesen eines nichtfiktionalen Textes in einem Punkt wesentlich unterscheidet: Der Leser nimmt Anteil am Schicksal der Protagonistin, wobei er entscheidet, ob er ihr Verhalten nachvollziehen kann und, wenn das der Fall ist, ob er ihr z. B. Verständnis oder Ablehnung entgegenbringt: „Dramatisch ausgedrückt, ob er sie bewundert oder verabscheut“ (Bredella 2012, 5).

Eine Besonderheit beim Lesen eines literarischen Textes ist also, dass der Leser dem Text nicht nur Informationen entnimmt, sondern diese auch bewertet, was Bredella als einen dialogischen Prozess bezeichnet, welcher für die Entwicklung von Urteilsfähigkeit und Empathiefähigkeit ausschlaggebend ist (Bredella 2012, 5). Der Leser ist bei dem dialogischen Prozess als Beobachter des Geschehens zu verstehen, der sich für die Parteinahme von A oder B entscheidet. Freilich kann man sich auch in nur eine leidende Figur einfühlen, aber als Beobachter bedenkt man den Verursacher des Leids mit und entscheidet sich so für das Opfer der imaginären Konfliktsituation, so dass man auch in diesem Fall von einem dialogischen Prozess sprechen kann. Dem hier zur Anwendung kommenden Empathieverständnis liegt eine funktionale Beziehung von Kognition und Emotion zu Grunde, welche in einem nicht voneinander trennbaren Mischungsverhältnis auftritt (Olsen 2011, 4).

Die Fähigkeiten Emotionen wahrzunehmen und Empathie zu entwickeln wurden in der Deutschdidaktik früher unter dem Begriff Fremdverstehen bzw. ,Verstehen' subsumiert. Dabei ist die Dichotomie von ,eigen' und, fremd' problematisch bzw. aus heutiger Sicht missverständlich oder unzeitgemäß. Olsen $(2011,11 \mathrm{f}$.) hält diese Begriffe aus einem weiteren Grund für nicht vertretbar, da sie zu einseitig kognitiv ausgerichtet seien. Er schlägt deshalb vor, die Teilkompetenzen Emotionsverstehen, was eine kognitiv orientierte Fähigkeit ist, und Emotionseinnahme, was eine stärker emotional orientierte aktive Tätigkeit ist, unter der weniger kontrovers diskutierten literaturwissenschaftlich orientierten Wendung ,literarische Kommunikation“" zu subsumieren. Inwieweit der Verständnisbegriff im norwegischen Lehrplan ebenfalls zu einseitig kognitiv ausgerichtet ist und auf der mittlerweile überholten Annahme, dass emotionale und kognitive Vorgänge zu trennen sind, beruht, kann hier nicht näher diskutiert werden.

Der aus früheren Lehrplänen übernommene Toleranzbegriff im norwegischen Lehrplan LK-06, wurde von Afdal (2006) eingehend untersucht. Afdal unterstreicht hierbei, dass es eine unüberschaubare Anzahl theoretischer Arbeiten und Schriften zum Thema Toleranz gibt, wobei nur Einigkeit darüber herrscht, dass wenig Konsens besteht. Demzufolge kann es letztlich nicht nur eine Definition von Toleranz geben, sondern sie muss eine gewisse Breite haben. Den Toleranzbegriff im norwegischen Lehrplan kann man als ein ,dichtes“ Konzept im Geertz'schen Sinne bezeichnen, welches ein breites Spektrum von Phänomenen und deren Beziehung untereinander miteinbezieht. Dieser weitgefasste Begriff umfasst ein Konzept, das man so entgrenzen kann, dass es ,[1]iving peacefully with difference“" (Afdal 2006, 15) bedeutet. Dabei gibt es Überschneidungen mit anderen verwandten Begriffen wie Respekt, intellektueller Freiheit und Verständnis. Afdals Untersuchung begrenzt sich auf die Grundschule und Sekundarstufe I (Klasse 1-10) und zeigt, wie der im Lehrplan intendierte Toleranzbegriff wahrgenommen und von Lehrern interpretiert wird. Anhand des hier vorgestellten Unterrichtskonzepts soll u. a. konkretisiert werden, wie man sich im 
Fremdsprachenunterricht mit den Themen „Toleranz“ und „Verständnis“ auseinandersetzen kann, und wie die Lerner damit umgegangen sind.

Selbst wenn die Lehrplanbegriffe „Verständnis“ und „Toleranz“ tendenziell eher kognitive Fähigkeiten benennen, bleibt die übergeordnete Fragestellung, in welchem Maße Leser Emotionen von Figuren erfassen können und inwieweit sie (kurzzeitig) die dargestellten Emotionen übernehmen.

Zur Analyse der Ergebnisse aus der Unterrichtsbeobachtung, den Lernerarbeiten und dem Fragebogen werden die von Olsen entwickelten Niveaustufen literarischer Empathiefähigkeit (Olsen 2011, 11f.) herangezogen. Hierzu soll je eine Teildimension für die Emotionswahrnehmung (Niveaustufe A-E) und Emotionseinnahme (Niveaustufe A-D) fruchtbar gemacht werden:

\section{Teildimension1: Emotionswahrnehmung}

$1 \mathrm{E}$ Ein Leser ist in der Lage, verschiedene emotionale Zustände von Figuren auf spezifische Hintergründe (zum Beispiel Ideologien, historisch/biographische Bedingungen etc.) zu beziehen.

Teildimension 2: Emotionseinnahme

2C Ein Leser ist in der Lage, sich verschiedene emotionale Zustände einer oder mehrerer Figuren, die in einem literarischen Text dargestellt sind, zu vergegenwärtigen. Er kann auch (mögliche/plausible) Gründe, die im Inneren der Figur/en liegen, für die erfassten emotionalen Zustände nennen, da er kurzzeitig aktiv die jeweiligen Zustände einnimmt (Olsen 2011, 11-12).

In diesem Zusammenhang könnte man sich fragen, wie eine fiktionale Geschichte Wahrheitsbzw. Erkenntnisanspruch erheben kann, bzw. wie man u. a. eine Brücke von der Empathie, die die Lernenden für eine nicht reale, fiktionale Figur entwickeln, zur Empathiefähigkeit in der realen Lebenswelt schlagen kann.

Der Anthropologe Victor Turner (1988) spricht von einem magischen Spiegel, den uns alle Geschichten vorhalten: ,they exaggerate, invert, re-form, magnify, minimize, dis-color, re-color, even deliberatly falsify, chronicled events" (Turner 1988, 42). Bredella betont, dass dadurch, dass es sich um einen magischen Spiegel handelt, eine zentrale Einsicht zum Ausdruck kommt, nämlich, dass Verfremdung notwendig ist, um sichtbar zu machen, was sonst unsichtbar bleibt. Empathie für eine fiktionale Figur entsteht erst, wenn sich der Leser bewusst emotional engagiert: „Erst wenn und weil der Leser/Hörer/Erzähler sich für den einen und nicht den anderen entschieden hat, nimmt er Anteil, konstruiert dessen Geschichte, narrativiert" (Breithaupt 2009, 171). Empathie entsteht u. a. dann, wenn der Leser/Bilderbuchbetrachter Menschen in Entscheidungssituationen antrifft und Vermutungen über deren Handeln anstellt: „,Diese Tätigkeit des Mitratens, Mitüberlegens, Mitreagierens und Auskundschaftens der möglichen und unmöglichen Möglichkeiten knüpft uns an den Beobachteten. Ohne dieses Mitraten und Vorhersagen gäbe es wohl keine ,Empathie““ (Breithaupt 2009, 77f.). Wichtig für den Zusammenhang des Unterrichtsprojekts ist, dass das Handeln der Figuren vom Leser bewertet wird, dass also während des komplexen Lesevorgangs Verständnis und Toleranz für die Figuren entwickelt werden können, was wiederum in der Lebenswelt der Lernenden fruchtbar gemacht werden kann. Geschichten 
ermöglichen, dass man sich kognitiv und emotional gefahrlos auf neue Welten einlassen und in ihnen, im Unterschied zum realen Leben, probeweise handeln kann (Bredella 2012, 12).

Im Unterrichtszusammenhang würde es nicht genügen, einen Text lediglich danach auszusuchen, ob er Verständnis und Toleranz ermöglicht. Beides muss mit anderen Lernzielen eine funktionale Verbindung eingehen. Dabei wird von der Hypothese ausgegangen, dass es ohne Empathie nur schwer gelingen kann, die weiteren Lernziele des eingangs zitierten Lehrplans für den Fremdsprachenunterricht zu erreichen.

\section{Geschichte, von unten'}

Geschichte kann man etwas vereinfacht ausgedrückt, von oben` aus der Sicht der Herrschenden oder, von unten', aus der Sicht der Beherrschten bzw. der ,kleinen Leute“ vermitteln. Geschichtsvermittlung, von unten' ist in der Literaturgeschichte nichts Neues. Ein bekanntes Beispiel aus der Literatur ist das Gedicht „Fragen eines lesenden Arbeiters“ von Bertolt Brecht, das 1936 im Exil entstanden ist (Brecht 2010, 74). Brecht ist weder der Einzige noch der Erste, der sich mit Geschichte ,von unten' auseinandergesetzt hat. Brechts Gedicht eignet sich jedoch für die Vertiefung dieser Thematik im fremdsprachlichen Deutschunterricht in besonderem Maße, da es von einer bestechenden Einfachheit, Klarheit und optischen Materialität ist. Das Gedicht kritisiert die traditionelle Geschichtsschreibung, in der nur die Namen der ,Großen', d.h. der Könige, der Herrscher, der Sieger, verzeichnet sind. Der lesende Arbeiter will u. a. wissen, wer das sagenhafte Theben, das mehrmals zerstörte Babylon und die Stadt Rom mit ihren zahlreichen Triumphbögen erbaut hat. Aus der Perspektive eines Arbeiters stellt er Fragen zur Geschichte bzw. zur gängigen Geschichtsschreibung und konstatiert, dass in den Geschichtsbüchern beinahe ausschließlich über Kaiser und Könige berichtet wird, weil die Geschichtsbücher aus der Sicht der Herrschenden geschrieben wurden. Aber er erkennt Zusammenhänge. Herrscher und Beherrschte sind als gleichwertig aufzufassen. Das Gedicht endet zur kritischen Hinterfragung dieser Feststellung mit zwei nahezu parallelen Zeilen, die nacheinander gesetzt sind: „So viele Berichte. / So viele Fragen.“ (Brecht 2010, 74)

Mit diesen Versen deutet der nur scheinbar naive Arbeiter an, dass jeder Bericht, von oben' genauso viele Fragen, von unten' provoziert. Bei Brecht wird der soziologische Aspekt der Geschichtsschreibung Gegenstand des Gedichts. Die Hierarchie von ,oben` und ,unten ‘ wird in der Zeilenanordnung sinnfällig, d.h. die optische Materialität ist beabsichtigt und sollte im Unterricht thematisiert werden.

Brechts marxistische Perspektive wird im Bilderbuch Die Lisa um einen Genderaspekt erweitert, es handelt sich um die Perspektive ,von unten“ und von einer Frau. Man könnte vielleicht sagen, dass die Hauptfigur in doppelter Hinsicht benachteiligt ist, sowohl durch ihre soziale Stellung als auch durch ihr Geschlecht. Im Zusammenhang mit der Thematisierung der Genderperspektive wurde im Unterricht u. a. auf das Wahlrecht für Frauen eingegangen, das in Norwegen einige Jahre früher als in Deutschland durchgesetzt wurde.

Seit Ende der 1980er Jahre ist es in Deutschland in den Geschichtswissenschaften zu einer Neubewertung fiktionaler Texte und zu einer Renaissance der Unterrichtsmethode der Geschichtserzählung gekommen. Die ,neuen' geschichtsdidaktischen Unterrichtsmodelle sind ganz entscheidend durch Konzepte aus dem Deutschunterricht und der Arbeit mit fiktiven Texten beeinflusst worden, denn es steht außer Zweifel, dass fiktive Texte bei ihren Lesern jene Imaginationskraft freisetzen, ohne die das historische Lernen nicht möglich wäre. Zimmermann (2004) betont, dass die Verwendung eines fiktionalen Textes zum Zweck 
historischen Lernens nur legitim und sinnvoll ist, wenn der Leser dabei in die Lage versetzt wird, zwischen faktualen und fiktionalen Texten zu unterscheiden.

Die Lisa, 1991 zum ersten Mal herausgegeben, steht in dieser Tradition der

Geschichtserzählung, wobei für die Verwendung in der deutschen Grundschule auch ein Begleitheft (Meisenberg 2004) ausgearbeitet wurde. Das Begleitmaterial enthält $u$. a. eine Text-Bild-Synopse, die den Lernenden einen ersten Überblick über die in sechs Zeitabschnitte gegliederte Handlung des Buches gibt und Karteikarten, die einzelne Stichworte vertiefen. Weiteres Vertiefungsmaterial und weitere Arbeitsaufgaben (siehe Anhang Anlage 2) wurden neu formuliert um das Buch einer älteren Lernergruppe anzupassen.

\section{Kurze Zusammenfassung der Handlung, Zeitabschnitte und Bilder}

Die Hauptfigur Lisa wird 1899 in Berlin im Wiesenweg 10 in einer Wohnung in einem Stadthaus geboren, so wie sie vor der Jahrhundertwende häufig auf Wiesen errichtet wurden und sich zu Häuserzeilen in den rasch wachsenden Großstädten entwickelt haben. Die Hauptfigur wohnt ihr ganzes Leben im selben Haus, in derselben Straße, die aber mehrmals ihren Namen wechselt. An den Namensänderungen kann man nicht nur die Stadtentwicklung, sondern auch die politische Entwicklung ablesen, wovon die Hauptfigur nicht unbehelligt bleibt. Die Protagonistin arbeitet als Schneiderin und gehört zu den einfachen Leuten, was auch durch die Namensgebung, die Lisa' deutlich wird, was so viel wie das einfache Mädchen von neben an bedeutet. Am Ende des Textes, etwa um 1990, ist die Protagonistin über 90 Jahre alt und hat Weltgeschichte erlebt, die sich sozusagen vor ihrer Haustür abgespielt hat.

Die deutsche Geschichte des 20. Jahrhunderts auf nur ca. 30 Seiten in bzw. mit wenigen Zeilen zusammenzufassen und auf einen Handlungsort zu reduzieren, muss zwangsläufig zu Verkürzungen und Vereinfachungen führen. Entscheidend für die Verwendung im Unterricht ist, dass die, große“ Geschichte mit der ,kleinen“ Geschichte verknüpft wird und dass an der Biografie der Hauptfigur gezeigt wird, was viele erfahren haben. Die Zeitleiste wird im Text entwickelt und durch sechs Zeitabschnitte abgesteckt (Meisenberg 2004, 3-6): ${ }^{1}$

1: Die Kaiserzeit bis zum Ende des Ersten Weltkrieges/Revolution (1899-1919) S. 3-9.

2: Die Weimarer Republik (1919-1933) S. 10-13.

3: Die Nazi-Diktatur (1933-1945) S. 14-19.

4: Vom Kriegsende bis zum Wiederaufbau (1946-1955) S. 20-25.

5: Das geteilte Deutschland BRD und DDR (1958-1967) S. 26-31.

6: Deutschland bis zur Wende (1970-1990) S. 26-31.

Diese sechs Zeitabschnitte bilden den Ausgangspunkt zur Einteilung der Lernenden in sechs Arbeitsgruppen. Der Erzähler des literarischen Textes steht außerhalb des Geschehens und besitzt die Übersicht. Dem französischen Erzähltheoretiker Genette zufolge handelt es sich um einen extradiegetischen Erzähler mit Nullfokalisierung (vgl. Martinez und Scheffel 2009,

\footnotetext{
${ }^{1}$ Die folgenden Seitenangaben beziehen sich auf Klaus Kordon/Peter Schimmel. Die Lisa Eine deutsche Geschichte. Weinheim: Beltz, 2002/2007. Die Seitenangaben im Begleitheft weichen von den Seitenangaben im Bilderbuch ab und sind hier korrigiert. Die Angaben zitieren die Kapitelüberschriften.
} 
188f.). Erzähltempus ist das Präteritum, bis auf Lisas letzte Lebensphase, die im Präsens erzählt wird. Die Bilder repräsentieren markante Momente im Leben der Protagonistin. Die wenigen Textzeilen unter jedem Bild sind in einem sachlich-nüchternen Ton gehalten, wobei die einzelnen Sätze kurz sind und oft lapidar anmuten. Aber gerade dieser Tonfall, die Auswahl prägnanter Momente und deren knappe Darstellung können dem Leser ,,unter die Haut gehen“" (Brandstätter 2008, 32). Die lakonische sprachliche Darstellung transportiert, so verstanden, eine wesentliche Botschaft, nämlich die Machtlosigkeit der Protagonistin den weltgeschichtlichen Ereignissen gegenüber. Diese Dimension wird in der Zusammenschau mit den Bildern vielleicht noch deutlicher.

Die 31 großformatigen Bilder, die zwei Drittel jeder Seite einnehmen, bestimmen das Aussehen des Buches. Die Bilder illustrieren nicht den Text, sondern übermitteln eigenständige Botschaften. Dadurch, dass die Bilder die Gefühle und Gedanken der Personen abbilden, die im Text weitgehend ausgespart sind, verlangen die Bilder vom Betrachter ein besonderes Sich-Einlassen.

Zunächst ist festzuhalten, dass die Bilder eine tendenziell cartoonhafte Gestaltung haben, wobei sie gleichzeitig äußerst detailgetreu sind. Die Bilder bestehen aus einer Mischung aus Aquarelltechnik und Zeichnung, was eine gewisse Unschärfe bewirkt, wodurch sie aber an Lebendigkeit gewinnen. Da es sich um eine Bilderfolge handelt kann man mit Begriffen aus der Filmsprache festhalten, dass es Frontal- und Panoramabilder sowie ein Bild aus der Vogelperspektive gibt. Die meisten Bilder sind aus einer gewissen Untersicht konzipiert, was der Perspektive bzw. Augenhöhe eines Kindes entspricht, denn es handelt sich hier um die Gattung Kinder- und Jugendliteratur (KJL). Das erste und das letzte Bild bilden einen visuellen Rahmen um den literarischen Text, wobei auf dem ersten Bild der Blick durch ein Fensterkreuz auf die neugeborene Lisa gelenkt wird, die auf dem Schlussbild aus demselben Fenster den Betrachter als Neunzigjährige anblickt.

Der Betrachter sieht aber nicht einfach ein Bild, er sieht etwas im Bild. Bilder sind ein Medium des Sehens und gleichzeitig am Sehen selbst beteiligt. Bei der Betrachtung von Bildern findet eine Verkettung von und zwischen den Bildern statt, wobei Übergänge zwischen Sichtbarkeit und Nicht-Dargestelltem in den Blick geraten. Die Bilder präsentieren mit wenigen Ausnahmen eine Episode aus dem Leben der Hauptfigur, wobei die Geschichte des Hauses und seiner Straße ebenfalls thematisiert werden. Die Bilder erzählen dabei auch von der jeweiligen Staatsmacht, wobei die Wahl der Perspektivierung, von unten' nicht nur die Sicht eines Kindes, sondern vielleicht auch die Machtlosigkeit der relativ einfachen Protagonistin verstärken können. Diese erzähldramaturgische Sicht auf die Hauptfigur betont die Nähe zwischen Bilderbuch und Film, wobei die besondere ästhetische Kraft des Bilderbuchs im eigentümlichen produktiven Spannungsverhältnis von Bild und Text liegt. Laut dem Bilderbuchforscher Jens Thiele (2003) sollte eine Theorie von Bild und Text nach den fantasiestiftenden Momenten zwischen beiden Darstellungsformen, nach den Bildern im Kopf forschen und die komplexen Wechselwirkungen des offenen Bezugssystems berücksichtigen.

Das Füllen von Leerstellen zwischen Text und Bild und das Entdecken der Zusammenhänge zwischen den Bildern kann zur Einübung von Empathie und Toleranz beitragen, worauf in der Analyse der Lernerarbeiten vertiefend eingegangen wird.

Um die Mehrdeutigkeit der Bilder angemessen nutzen zu können, brauchen die Lernenden Zeit und eine gezielte Hinführung auf bildnerischer und sprachlicher Ebene. Die Fokussierung der Bilder, also die Wichtigkeit eines ,konzentrierten Schauens“ (vgl. Weidemann, 1988, 118) ist dabei hervorzuheben. Bildverstehen ist aus psychologischer Sicht 
jedoch ein Vorgang einer Vereinfachung oder Reduktion. Mit anderen Worten betrachtet ein Rezipient ein Bild nur so lange, bis er meint, das Bild verstanden zu haben. Das geht relativ schnell und ist weitgehend automatisiert. Es gibt aber noch ein anderes Bildverstehen, das sogenannte indikatorische Bildverstehen (vgl. Weidenmann, 1988, 118) das einsetzt, wenn die oberflächliche Informationsverarbeitung nicht ausreicht und man vielleicht wissen möchte, warum etwas so und nicht anders dargestellt ist. Wenn man also die Absichten hinter einem Bild verstehen möchte, braucht man mehr Zeit und muss genauer hinschauen. In diesem Zusammenhang liegt die Frage auf der Hand, ob die Lernenden dieses Feld nicht längst beherrschen. Inzwischen ist eine Bildschriftlichkeit entstanden, wobei auf Facebook, Instagram und Snapchat Bild und Text eine Art narrative Symbiose eingehen. Auf Snapchat sind Bild und Text nur für eine relativ kurze Zeit verfügbar und verlangen geradezu ein schnelles Bildverstehen.

Wenn im fremdsprachlichen Deutschunterricht mit einem Bilderbuch gearbeitet wird, sollen die Illustrationen $u$. a. als semiotische und ästhetische Ressource verwendet werden. Dabei ist zu vermeiden, dass die Lernenden die Bilder mit ihrem automatisierten Bildverstehen auffassen und sich schnell zum nächsten Bild weiterbewegen. Weiter ist die Verbindung zwischen Sprache und Bild offensichtlich und erwünscht, denn die volle Bedeutung und Interpretation wird erst durch und in Sprache entfaltet. Die Spezifika der beiden Medien sollen in Analyse und in Produktion miteinander verknüpft werden, indem Bilder zu Texten und Texte zu Bildern produziert und die Arbeits- und Erkenntnisprozesse reflektiert werden. Durch die mediale Transformation verändern sich die Kommunikation und ihre Intensität. Demzufolge erweitert die visuelle Kompetenz die kommunikativen Fertigkeiten, die im Fremdsprachenunterricht vermittelt werden (vgl. Dammmann-Thedens und Michalak 2012). Wie die Lerner mit Sprache und Bild in der Auseinandersetzung mit dem Bilderbuch vorgegangen sind, wird an einer Gruppenarbeit aus dem Hochschulbereich und an zwei Gruppenarbeiten aus dem Sekundarbereich im Abschnitt Realisierung der kreativ-produktiven Aufgaben vorgestellt.

\section{Methode}

Im Rahmen einer qualitativen Untersuchung wurde als Methode eine Fallstudie gewählt. Eine Fallstudie schließt nach Creswell (2007) sowohl wissenschaftliche Beobachtung (Observation), Fragebogen sowie schriftliche und visuelle Dokumentation mit ein und kann durch die Datenvielfalt zu einem tieferen Verständnis der zu erforschenden Phänomene beitragen. In der Doppelrolle als Lehrer und Forscher sind die unterschiedlichen Perspektiven als teilnehmender Lehrer und als distanzierter Forscher einzunehmen, wodurch zwei unterschiedliche Zugänge zur Interpretation der Daten erhalten werden (Hoel 1997; Postholm/Moen 2009).

Um die eingangs genannten Lernziele bzw. Phänomene Verständnis und Toleranz näher in den Blick zu bekommen, werden mittels ,dichter Beschreibungen“ im Geertz'schen Sinne die jeweilige Arbeitsweise beider Lernergruppen im Literatur- bzw. fremdsprachlichen Deutschunterricht festgehalten, deren Präsentationen ausgewertet und ein strukturierter Fragebogen, der im Anschluss an die Aufgaben ausgeteilt wurde, analysiert. Diese Studie ist auf die Arbeiten von 20 Studenten beschränkt, die sich in Kleingruppen über zwei Wochen im Rahmen von insgesamt vier 90-minütigen Unterrichtseinheiten mit dem Bilderbuch auseinandergesetzt haben, wobei in der letzten Unterrichtseinheit die Präsentationen 
vorgestellt wurden. Die zweite Lernergruppe besteht aus 25 Schülerinnen und Schülern der Sekundarstufe II, die in einem vierwöchigen Schulprojekt mit dem Bilderbuch gearbeitet haben.

\section{Kreativ-produktive Aufgaben}

Zum Bilderbuch wurden drei Arbeitsaufgaben erstellt, wobei hier nur auf die letzte, die produktiv-kreative Aufgabe eingegangen wird (vollständige Aufgabenstellung, s. Anhang 1). Diese Aufgabe besteht aus drei Teilen, einer Bildbeschreibung aus dem von der jeweiligen Gruppe gewählten Zeitabschnitt, einem Tagebucheintrag und einer kreativen Aufgabe, bei der auch andere Medien benutzt werden sollen. Im Umgang mit erzählenden Texten sind produktive Verfahren, wie Briefe von und an Figuren zu schreiben bzw. Tagebucheinträge zu schreiben (vgl. Spinner 2005, 986; Ehlers 2016, 172-174), sinnvoll, weil damit Gedanken und Erlebnisweisen von Figuren zum Ausdruck gebracht oder Stellungnahmen zum Verhalten von Figuren abgegeben werden. Somit wird ebenfalls der Absicht Rechnung getragen, die Lerner dazu zu veranlassen, sich anhand der Perspektive der Hauptfigur in den gewählten Zeitabschnitt hineinzuversetzen.

\section{Präsentation der kreativ- produktiven Aufgabe}

Aufgabe 3: Diese Aufgabe besteht aus drei Teilen

Bildbeschreibung: Wählen Sie ein oder zwei Bilder aus der von Ihnen zu bearbeitenden Lebensphase von Lisa aus und beantworten Sie die Fragen hierzu. Tipp: Benutzen Sie hierzu das Internet.

Tagebucheintrag: Wählen Sie eine Episode aus der von Ihnen gewählten Lebensphase von Lisa aus und schreiben Sie einen Tagebucheintrag.

Kreative Aufgabe: Wählen Sie eine passende Überschrift zu Ihrer Lebensphase von Lisa. Schreiben Sie z. B. einen Dialog zu einer Begebenheit aus Lisas Lebensphase. Gestalten Sie dazu ein Bild. Nutzen Sie hierzu u. a. die Möglichkeiten im Netz. (Bitte legen Sie eine Liste Ihrer Quellen an, die Sie separat abgeben.)

Nachdem die Arbeitsaufgaben präsentiert wurden und die Lerner sich in verschiedenen Räumen in Gruppen zusammengesetzt haben, sucht der Lehrer die Lernergruppen auf, erkundigt sich nach deren Fortschritten und bietet gegebenenfalls Hilfe an.

\section{Analyse ausgewählter kreativ-produktiver Arbeiten}

Voraussetzung für die Analyse der Lernerarbeiten ist, dass die Lehrkraft eine reflektierende und untersuchende Haltung gegenüber dem eigenen Unterricht einnimmt und in der dialogischen Auseinandersetzung mit den Rollen als Lehrkraft und ,Praxisforscher unterschiedliche Interpretationszugänge fruchtbar macht, um die Wirkung des Unterrichtskonzepts zu überprüfen (Riemer 2010). Anhand der vorgestellten Lernerarbeiten aus dem Hochschul- und Schulbereich soll gezeigt werden, wie die Lernenden die beiden bereits genannten Niveaustufen literarischer Empathiefähigkeit entwickelt haben. Eine ,dichte Beschreibung' aus der ersten Seminarstunde kann dies verdeutlichen. Ein Student aus einer Dreiergruppe, die die Zeit des Nationalsozialismus (1933-1945) bearbeitet (Kordon/Schimmel 2001, 14-19) berichtet, dass sie sich innerhalb der Gruppe gefragt haben, wo die Lisa gewesen 
sein könnte, als ihre Freundin Else von der Gestapo abgeholt wurde (Abbildung in Kordon/Schimmel 2001, 19):

Die Lisa ist ja nicht gerade eine Sympathieträgerin. Wie sollen wir sie schildern, sie hilft ihrer jüdischen Freundin nicht, sie sieht tatenlos zu, als Else abgeholt wird. Aber Lisa ist die Hauptfigur in der Geschichte, allzu schlecht wollen wir sie auch nicht darstellen. Wenn wir sie der Else gegenüber zu hilfsbereit dargestellt hätten, wäre sie vielleicht auch deportiert worden, dann wäre das ein anderes Buch geworden. Im Tagebuch wollen wir schreiben, dass Lisa Angst hat, man täte ihren Kindern etwas an, wenn sie Else helfen würde.

Abschließend sagt ein anderer Student in dieser Gruppe: „Die Lisa ist emphatisch, steht aber sich selbst am nächsten." Die Gruppe fertigt eine Powerpointpräsentation mit der Überschrift „Die braunen Jahre“ an und im Tagebucheintrag (Abbildung 1), der hier im Originaltext, d.h. ohne Korrekturen wiedergegeben wird, schreibt die Gruppe:

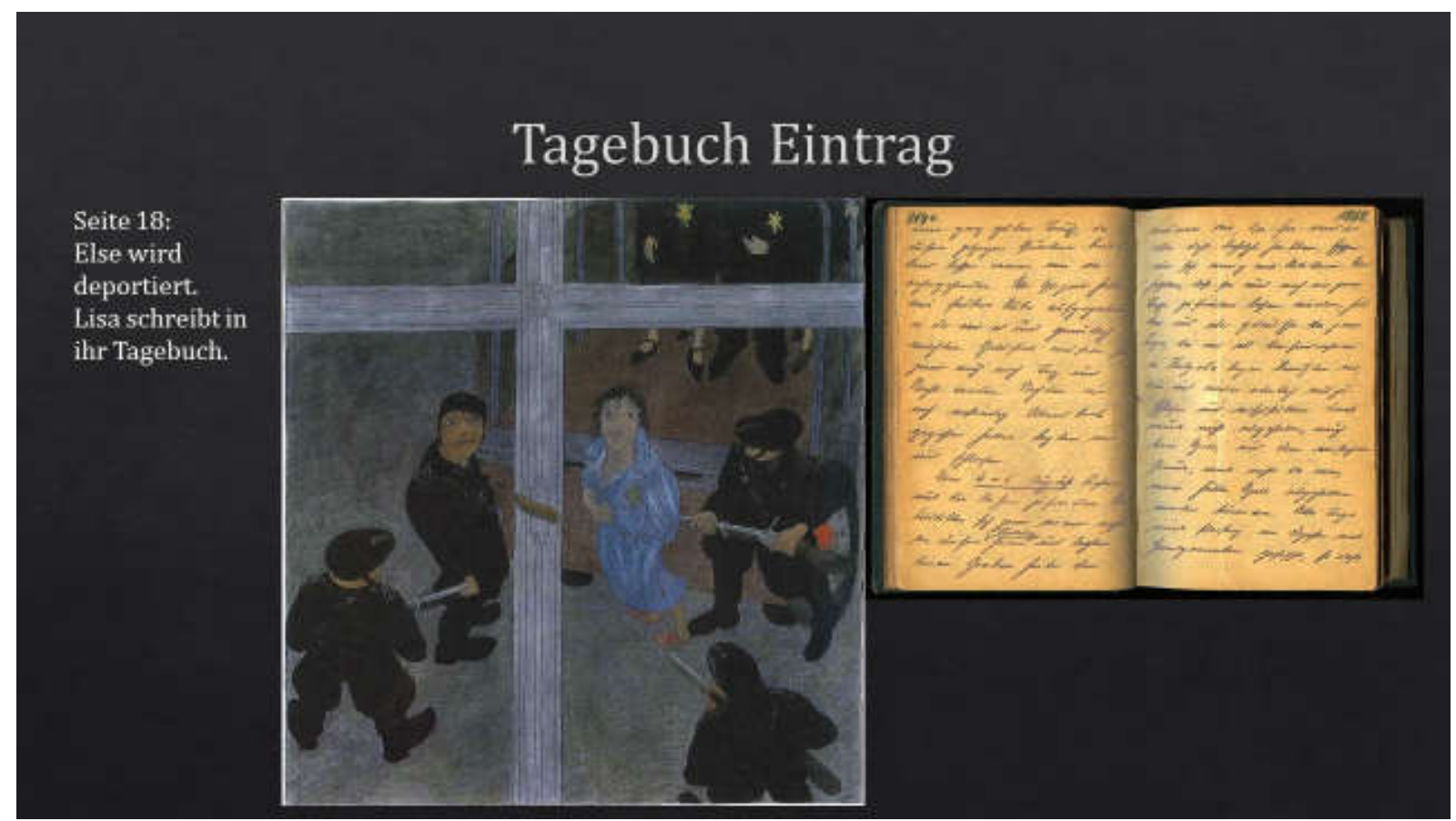

\section{Abbildung 1 DIE BRAUNEN JAHRE-TAGEBUCHEINTRAG}

Tagebuch Eintrag, den 14. Juli 1942

[...] Else kam nachmittags mit einem Brief vorbei. [...] sie muss nächste Woche vor der Polizeiwache mit einem Koffer erscheinen. Sie fragte unverfänglich danach, ob sie ein paar Wochen in unserem Dachboden wohnen könnte. Gerade da kam mein Klaus in die Küche und ich dachte daran, welche Folge mein Beistand für meine Kinder haben könnte. [...] Ich fürchte aber sehr, was mit meiner lieber Kindheitsfreundin geschehen werden. 
Bei der Entwicklung der Hauptfigur bezieht die Gruppe die historisch/ biographischen Bedingungen mit ein (vgl. Emotionswahrnehmung Niveaustufe 1E, Olsen, 2011) und beim Fortschreiben der Geschichte anhand eines Tagebucheintrags, führen sie plausible Gründe für das Verhalten der Hauptperson an (vgl. Emotionseinnahme 2C, Olsen 2011). Man kann also sagen, dass die Lerner Mutmaßungen über Lisas Charakter angestellt haben und ihr moralisches Dilemma in einer Entscheidungssituation bedacht haben. Wie bereits im Zusammenhang mit Breithaupts Überlegungen erwähnt, entsteht Empathie u. a. dann, wenn der Leser Figuren in Entscheidungssituationen antrifft, wo er Vermutungen über deren Handeln äußert, etwa ob Lisa Else, die in Lebensgefahr schwebt, auf dem Dachboden verstecken soll oder nicht. Die Lerner zeigen weiter, dass sie ihr geschichtliches Hintergrundwissen anwenden, wodurch sie tiefer in das Leben der Lisa eindringen und in einem dialogischen Prozess darüber entscheiden, dass sie für das Dilemma von Lisa ein gewisses Verständnis und ein gewisses Maß an Toleranz aufbringen möchten. Als kreative Aufgabe entwirft die Gruppe eine Feldpostkarte (Abbildung 2):

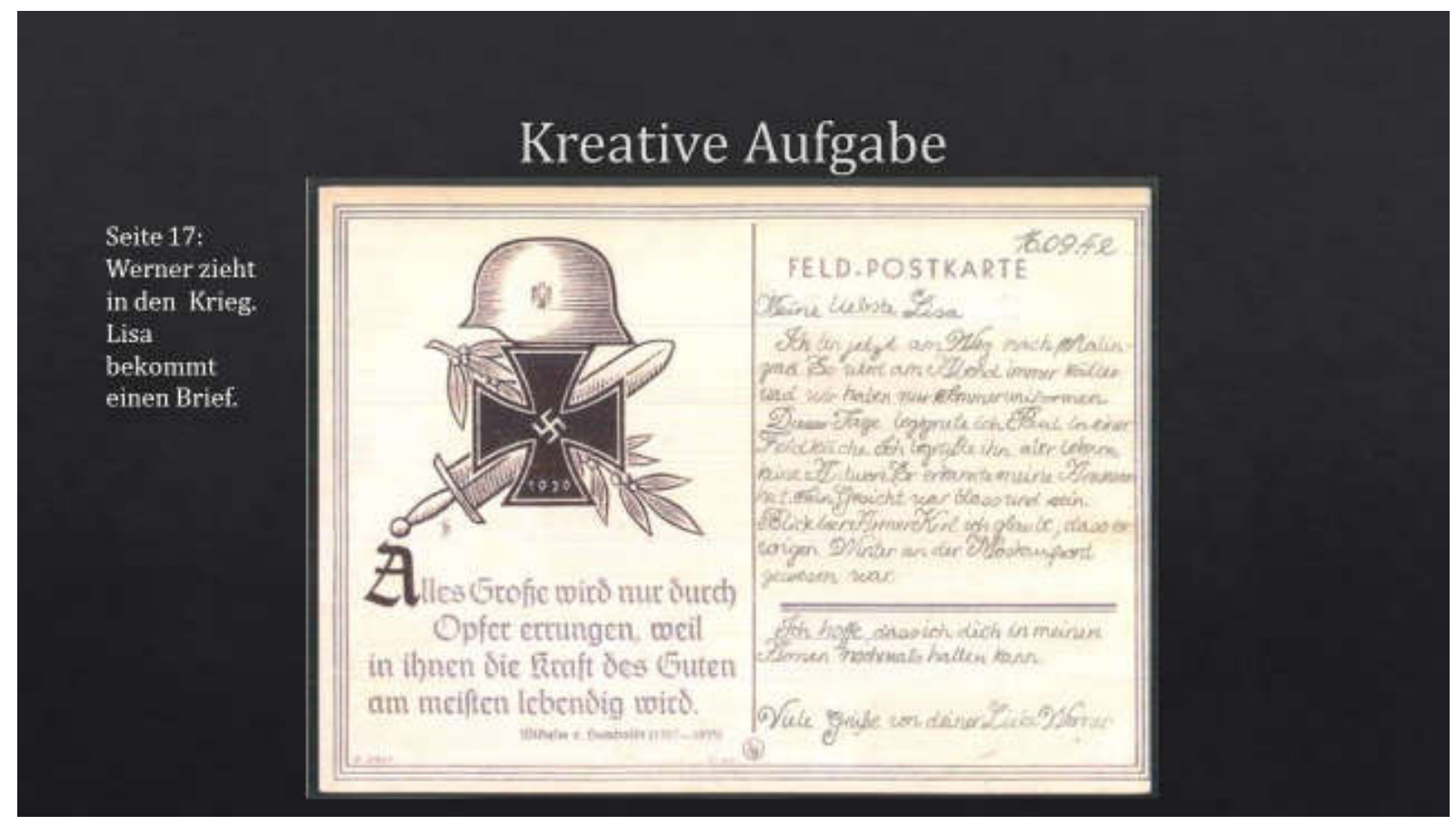

Abbildung 2 DIE BRAUNEN JAHRE- FELDPOSTKARTE

Meine liebste Lisa, 16.09 .42

Ich bin jetzt am Weg nach Stalingrad. Es wird am Abend immer kälter und wir haben nur Sommeruniformen. Dieser Tage begegnete ich Paul in einer Feldküche. Ich begrüßte ihn, aber bekam keine Antwort. Er erkannte meine Anwesenheit. Sein Gesicht war blass und sein Blick leer. Armer Kerl, ich glaube, dass er vorigen Winter an der Moskaufront gewesen war. Ich hoffe, dass ich dich in meinen Armen nochmals halten kann.

Viele Grüße von deiner Liebe Werner 
Hier verknüpft die Gruppe, die ,kleine‘ Geschichte, d.h. das Schicksal von Elses Freund Paul mit der ,großen' Geschichte. Die Idee zum Treffen von Werner und Paul haben die Studenten schon in der ersten Stunde der Gruppenarbeit. Ein Mitglied der Gruppe kommt zur Lehrkraft und fragt, ob die Gruppe den relativ freien Rahmen der kreativen Aufgabe korrekt verstanden habe und ein Treffen arrangieren dürfe. Der Student begründet das Vorhaben damit, dass er ein Buch über einen Soldaten gelesen habe, der in Stalingrad gewesen sei, was er Die Lisa zitierend miteinander in Beziehung brachte: ,,Als der Krieg ins dritte Jahr ging, musste auch Werner wieder ins Feld [...]. ' [Kordon/Schimmel 2001, 17] Deshalb musste er nach Stalingrad." Der Student drückt dies mit deutlichem Engagement in der Stimme aus und reagiert erfreut, als die Lehrkraft dem Vorschlag zustimmt.

Als der Student vorschlägt, sein Wissen über den Kriegsverlauf in Stalingrad produktiv zu verwenden, geht er von einem relativ kleinen Detail in Die Lisa aus, in das er sein Geschichtswissen hineinliest. Als Produkt fertigt einer der Studenten aus dem Original einer unbeschrifteten Feldpostkarte eine eigene beschriftete an, die Werner an Lisa schickt (Abb. 2). Die Worte auf der Feldpostkarte stellt er dabei mit einem schnörkelreichen, der deutschen Schrift nachempfundenen Stil dar. Der Text auf der Feldpostkarte gibt ebenfalls Auskunft darüber, dass sich diese Studentengruppe in fiktive Personen hineinversetzen und Empathie und Verständnis für deren Lage ausdrücken kann. Mitleid mit Paul kommt durch die Formulierung ,Armer Kerl` zum Ausdruck, wobei die unausgesprochenen Grausamkeiten an der ,Moskaufront` als Ursache für Pauls apathische Reaktion dem Freund gegenüber angerissen werden.

Die abschließende Präsentation der kreativen Aufgabe beginnt die Gruppe mit der Bildbeschreibung. Die Studenten wählen ein Panoramabild, das über zwei Buchseiten geht (vgl. Kordon/Schimmel, 14-15), wobei in der Mitte der Jugendfreund der Protagonistin, Karl Jäger in der für die Sturmabteilung (SA) typischen Uniform mit Schaftstiefeln, Koppel, Schulterriemen und Armbinde mit Hakenkreuz steht. Die Mitglieder der SA waren von einem völkisch-nationalsozialistischen Gedankengut geprägt und sind für zahlreiche Terroraktionen gegen Juden verantwortlich. Die Figur Karl Jäger hält ein Schild mit der Aufschrift „Deutsche! Wehrt Euch! Kauft nicht bei Juden!“ hoch. Zur Bildbeschreibung im Plenum haben die Studenten ein Stichwortblatt dabei, aus dem das nachfolgende Zitat stammt:

Was denkt Lisa? Dass sich die Gemeinschaft in der Straße auflöst. Sie waren (doch) alle Freunde in der Kindheit. Nun sind Deutsche gegen Deutsche, Nachbarn gegen Nachbarn, Freund gegen Freund. Was ist mit der glücklichen Zeit passiert? Was empfinden Herr Kohn und seine Tochter Else? Hass. Entfremdung von der Gesellschaft und ihrem Vaterland. Sind sie nicht auch Deutsche? Die Blicke der Kinder: Verwirrung. Warum sind die Menschen wütend auf „Tante“ Else? [Die Stichworte sind auf grammatische Fehler korrigiert, E. W.-J.)

Auch der Stichwortzettel für die Bildbeschreibung ist ein Ausdruck dafür, dass die Studenten Empathie und Verständnis für die Figuren in der Lisa-Geschichte entwickelt haben. Während der Präsentation der Bildbeschreibung, dem Lesen der Feldpostkarte und dem Tagebucheintrag, herrscht eine auffällig betroffene Stille im Unterrichtsraum und einige Mitstudenten reagieren sichtlich bewegt.

Den Studenten gelang es, eine historische Gegenwärtigkeit zu vermitteln, die u.a. durch den Ton des Vortrags und ihr Engagement während der Präsentation zum Ausdruck kam. Die daraus entstandene besondere Stimmung könnte man als eine unsichtbare und 
flüchtige Dimension einer ästhetischen, also wahrnehmungs- und darstellungsbezogenen, Erfahrung bezeichnen, welche auch vorsprachliche Dimensionen berührt. Das ist etwas, was im Rahmen dieser Untersuchung nur angerissen werden kann. Während des Projektunterrichts in der Sekundarstufe II konnten ebenfalls Besonderheiten der literarischen Kommunikation beobachtet werden, die zeigen, dass die Lerner sich sowohl in die Hauptperson hineinversetzen, ihre Stärken und Schwächen benennen und sie mit dem historischen Geschehen in Bezug setzen können (Niveaustufe 1 E und 2 C, Olsen 2011, 11f.). Eine Zweiergruppe, die sich für den Zeitraum Kaiserzeit- bis zum Ende des Ersten Weltkrieges/Revolution entschieden hat, wählt den Ausschnitt aus Die Lisa (vgl. Kordon/Schimmel 2001, 9-11), in dem die Hauptfigur ihren künftigen Mann kennenlernt und heiratet. Einer der beiden Lerner blättert in der Vorbereitungsphase zwischen den Seiten 9-11 hin und her und sagt zur Lehrkraft, dass das der Zeitraum sei, in dem die Protagonistin am glücklichsten sei. Mit Trauer in der Stimme fährt er fort, wie schnell das Glück zu Ende gehen und wie schrecklich alles enden könne. Bei der Recherche im Netz findet die Gruppe u. a. heraus, dass am 13. Juli 1918 ein Zeppelin in Berlin landete und für großes Aufsehen sorgte. An diesem Tag soll Lisa ihren Mann zum ersten Mal treffen, berichten die Gruppenmitglieder der Lehrkraft mit Begeisterung. Die Verknüpfung der Hauptfigur mit dem historischen Geschehen (Niveaustufe 1 E und 2 C, Olsen 2011, 11 f.) beschreibt die Zweiergruppe in einem Tagebucheintrag, den sie im Rahmen einer Powerpointpräsentation in der Klasse vorlesen (Abbildung 3) und der hier ohne Korrekturen widergegeben wird:

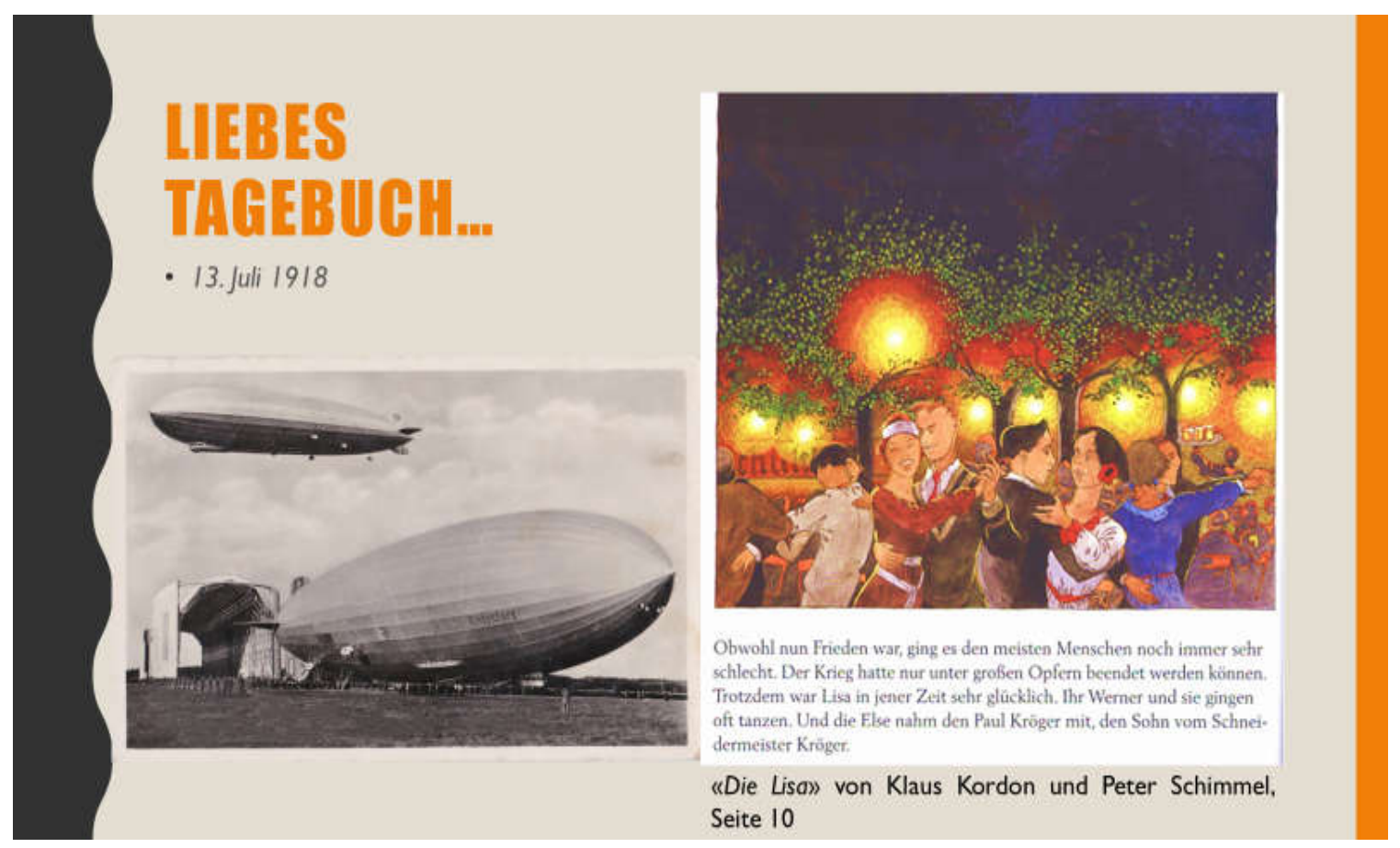

Abbildung 3 Tagebucheintrag Else lernt Werner kennen 


\section{Juli 1918}

Liebes Tagebuch,

Ich habe einen schneidigen Mann getroffen. Er hat blonde Haare und blaue Augen. Er wohnt in der Nähe in das Haus Nr. 17. Aber ich habe ihn nicht lange gekannt, weil er vor einigen Jahren von dem großen Krieg zurückgekommen ist. Einmal habe ich einkaufen gegangen. Plötzlich bin ich gestolpert und auf die Straße gefallen. Dabei habe ich meine Tüte verloren und alle meine Sachen sind auf die Straße gerollt. Ein junger Mann kam mir zu Hilfe und gepflückt die Waren hinauf, bevor ich etwas machen könnte. Der junge Mann hieß Werner. Heute Abend wollen wir in einem Park zu tanzen gehen. Es wird wie ein wahrgewordener Traum. Ich frage mich, was die Zukunft bringen wird?

Im nächsten Beispiel, in dem sich eine Dreiergruppe ebenfalls mit dem Zeitabschnitt Kaiserzeit bis zum Ende des Ersten Weltkrieg beschäftigt hat, wird die Verknüpfung der historischen Details mit der Protagonistin auf originelle Weise hergestellt. Die Lerner haben sich intensiv mit der Kaiserzeit bis zum Ende des Ersten Weltkrieges auseinandergesetzt und hierzu zahlreiche Quellen herangezogen. Sie fertigen eine Fotostory an, die sie in zwei Teile teilen, einen historischen und einen, der sich mit dem Bilderbuch und der Hauptfigur auseinandersetzt. Der historische Teil besteht u. a. aus Originalfotografien von Soldaten, Schützengräben, Landkarten und historischen Darstellungen der Verhandlungen am Kriegsende. Der Teil, der sich mit der Protagonistin im Bilderbuch auseinandersetzt, besteht aus selbständigen Interpretationen der Bilder von Peter Schimmel. Dabei ist der auffällige Kontrast zwischen den die Grausamkeit des Ersten Weltkrieges im Detail schildernden Schwarz-Weiß-Fotografien, die sich die Lerner herausgesucht haben, und den relativ einfach dargestellten farbigen Sockenfiguren beabsichtigt. Die Lerner teilen die Arbeiten untereinander auf, einer recherchiert im Netz und findet $u$. a. authentisches Bildmaterial aus der Zeit des Ersten Weltkriegs, ein anderer verfasst die zu schreibenden Texte, der Dritte fertigt die farbigen Illustrationen mit den Sockenfiguren an. Der Lerner, der die Sockenfiguren zeichnete, begründet die Bildgestaltung auf dem Fragebogen so: „Es war wichtig, die Charaktere auf eine humoristische Art darzustellen, um einen Kontrast zu dem sehr seriösen Thema zu erreichen“ (Übersetzung E. W.- J.). Auf den nachfolgenden Bildern wird die im Bilderbuch von Peter Schimmel gestaltete Scene der Adaption der Dreiergruppe gegenübergestellt: 


\section{Nordic Journal of Modern Language Methodology}

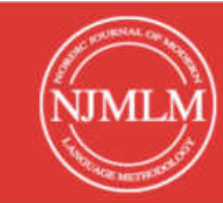

2018,6 (2), 3-27

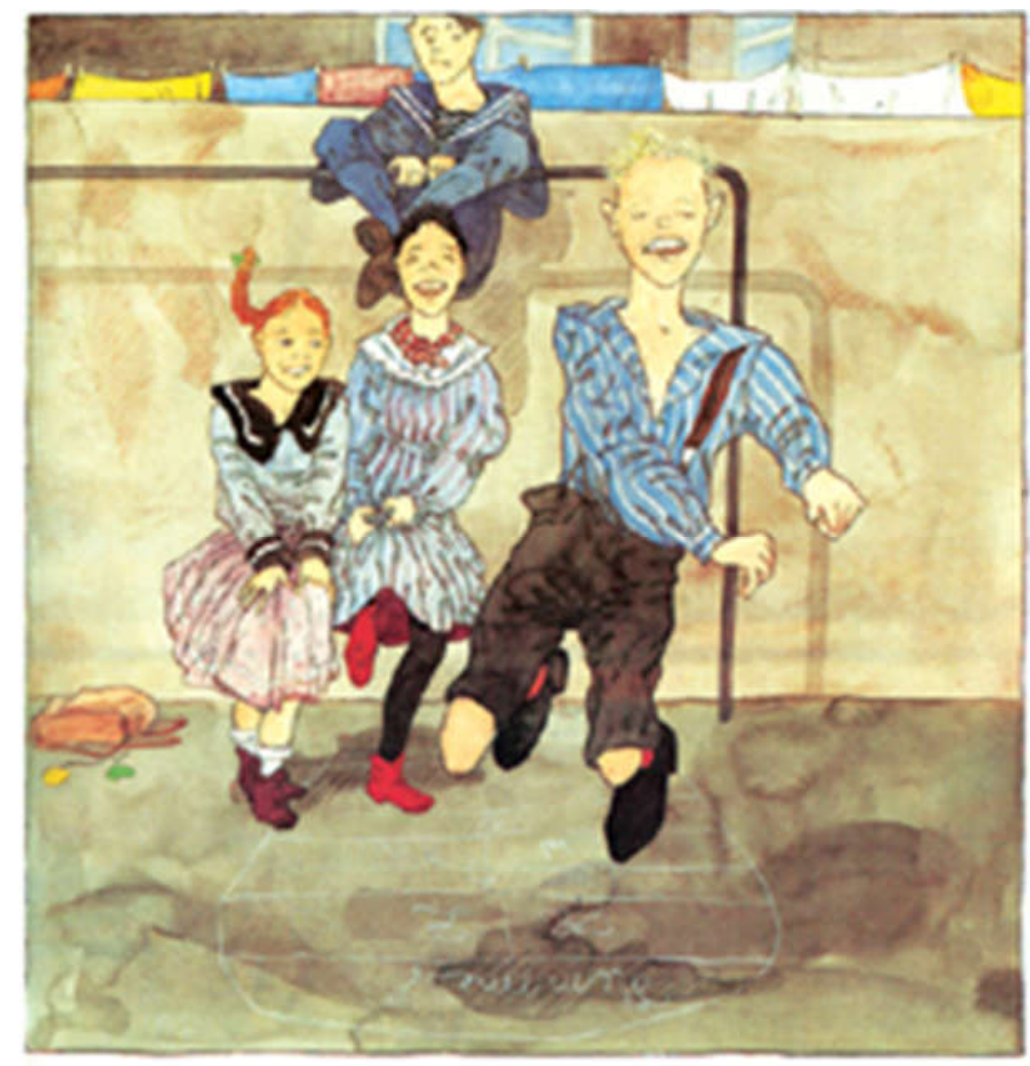

Abbildung 4 Die Lisa, S. 5 Illustration von Peter Schimmel

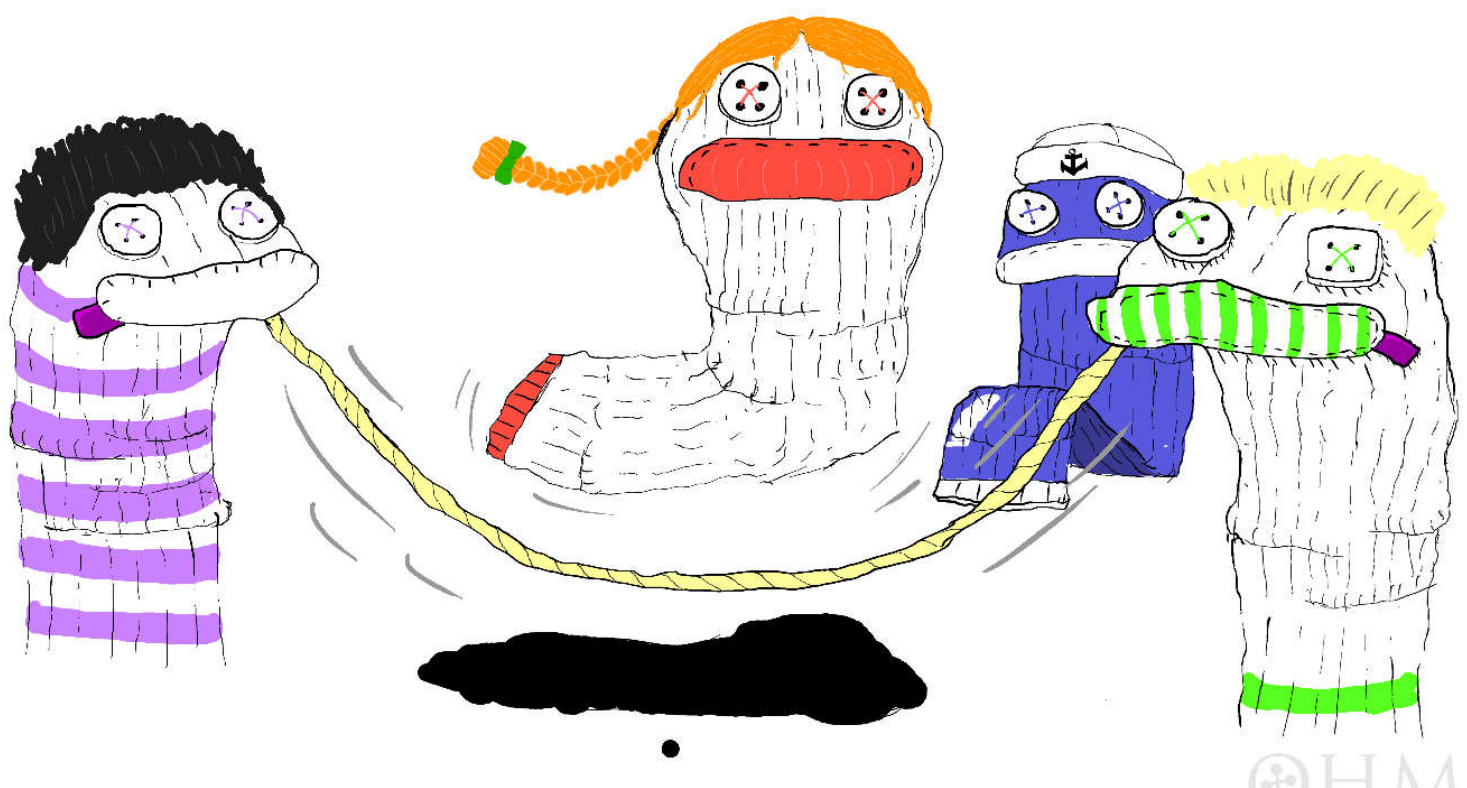

Abbildung 5 Illustration einer Lernergruppe 
In der Text-Bild-Synopse im Begleitmaterial zum Bilderbuch wird der Inhalt von Abbildung 4 so zusammengefasst: „Kinder tragen Matrosenanzüge, spielen Himmel und Hölle, Teppichstange, Schulranzen mit Schwämmchen“ (Meisenberg 2004, 6).

Auf der Illustration der Lernergruppe spielen die Kinder statt des Hüpfspiels ,Himmel und Hölle', Seilspringen, wobei die Hauptfigur mit ihrem charakteristischen Zopf als zentrale Figur in der Bildmitte zu erkennen ist. Aus der detaillierten Bildbeschreibung derselben Gruppe, die hier im Original, d.h. ohne Korrekturen widergegeben wird, geht hervor, dass die Gruppe sich in die im Bilderbuch abgebildeten Personen hineinversetzt hat und sich Zeit genommen hat, um die Bilder gründlich zu studieren. Eine verlangsamte Bildbetrachtung ist wie früher erwähnt für die Deutung des Dargestellten ausschlaggebend und wird vielleicht erst beim Lesen der gesamten Bildbeschreibung offensichtlich. ${ }^{2}$ So beschreibt die Gruppe das Doppelbild, das den Ausbruch des Ersten Weltkrieges thematisiert (vgl. Kordon/Schimmel 2002, 6-7):

\section{Bildbeschreibung}

[...] Dass Jahr ist 1914 und der Krieg hat genau begonnen. Im Mittelpunkt sehen Sie die Lisa, und sie steht dort mit ihrem Vater und ihrer Mutter. Es scheint, als ob sie einander sehr lieben. Wenn ein Krieg beginnt, erwartet man instinktiv traurige Gesichter und herzzerreißende Abschiede. Ganz im Gegenteil, gibt das Bild ein Eindruck von positive Gefühlen. Die Menschen lächeln und winken den Soldaten, die in den Krieg ziehen, mit ihren Hüten. Der Vater von Lisa scheint auch nicht traurig zu sein. Das macht das Bild ein bisschen sonderbar. [...] Die dominierende Farbe ist gelb, und das ist nicht nur auf dieser Bild. Auf allen Bildern während der Krieg gibt es immer gelben Himmel (Seite 6-9). Wir [...] haben in Norwegisch gelernt, dass gelb die Farbe der Fälschung ist. Das kann Sinn machen, weil es ganz falsch ist, was der Kaiser gesagt hatte. Der Krieg ging nicht zu Ende an Weinachten, und Deutschland konnte den Krieg nicht gewinnen. [...]

Bei der Auswertung des Fragebogens soll hier auf folgende Frage eingegangen werden (vgl. Anlage 3): Kannst du die Protagonistin (Hauptfigur) etwas näher beschreiben? Beschreibe u. a. was für eine Persönlichkeit sie hat. Wie zeigt sich ihre Persönlichkeit? Kannst du ihre Handlungen und ihre Reaktionsmuster verstehen? Diese Fragen beziehen sich auf das ganze Buch!

Bei den Antworten aus dem Sekundarbereich fällt auf, dass es den Lernern leichtfällt, die Hauptfigur zu charakterisieren, wobei sie sowohl Stärken als Schwächen der Figur benennen und Verständnis und eine gewisse Toleranz für das Verhalten der Hauptperson zeigen:

Ich verstehe, dass Lisa nicht besonders schlau/intellektuell ist. Aber sie kümmert sich um ihre Familie und die, die sie gernhat. Obwohl sie eine Gegnerin von unethischen und unmoralischen Handlungen ist, macht sie keine Anstalten zum Widerstand. Ich

\footnotetext{
${ }^{2}$ Die vollständige Bildbeschreibung befindet sich aus Platzgründen im Anhang (vgl. Anlage 2).
} 
würde sagen, sie ist handlungsschwach und etwas willensschwach. Mit Ausnahme von ihrem Haus, dort wollte sie ihr ganzes Leben wohnen bleiben. (Übersetzung E. W.-J.)

Ein weiterer Lerner antwortet auf den Auftrag eine Charakterisierung von Lisa vorzunehmen:

Sie wirkt wie eine Person, die dem Strom folgt, mit anderen Worten, sie ist nicht so individuell. Sie hat einen teilweise schwachen Charakter, der leicht von der Menge geleitet werden kann. Auf diese Art kann ich verstehen, dass sie viel auf Grund dessen handelt, wie sie handelt. (Übersetzung E. W.-J.)

Ein weiterer Lerner beantwortet den Charakterisierungsauftrag so:

[...] Sie liebt ihre Familie, was deutlich neben ihren Ansichten zum Ausdruck kommt (z.B. mag sie nicht, wie Karl Jäger zum Krieg beiträgt). Ich verstehe, wie sie handelt und in solchen Situationen reagiert, und ich würde sagen, das ist ziemlich normal für andere in derselben Situation.

\section{Schlussbemerkung}

Die übergeordnete Fragestellung dieser Untersuchung ist, wie die Auseinandersetzung mit der Lebensgeschichte der Hauptfigur zur Vertiefung der deutschen Geschichtskenntnisse und zur Entwicklung von Toleranz und Verständnis beitragen kann. Hierzu sollte an Beispielen aus der Unterrichtspraxis gezeigt werden, wie man mit dem Bilderbuch Die Lisa, kreativproduktiv arbeiten kann. Dabei sollten weniger die Komplexität reduzierenden Eigenschaften eines Bilderbuchs fokussiert werden, sondern etwas Anderes in den Vordergrund rücken. Es war mehrmals die Rede davon, dass die Bilder im Buch ganz eigenständige Botschaften übermitteln. In diesem Zusammenhang spielt die Vermittlung der Gefühle eine bedeutsame Rolle, die sich leicht an den Gesichtsausdrücken der Figuren ablesen lassen. Die Lerner bekommen durch die Bilder eine Möglichkeit die Reaktionen der Hauptfigur und der anderen Personen auf die Geschehnisse zu sehen und zu deuten, was ihnen dadurch, dass es keine sprachlichen Barrieren gibt, leichter fällt, als einen Text in der Fremdsprache zu erschließen. Die Antworten auf den Fragebögen bestätigen diese Annahme. Als Antwort auf die Frage, ob die Bilder in Die Lisa dazu beigetragen, dass die Lerner die Thematik besser verstehen und verankern können, schreibt ein Student auf Norwegisch etwa: „Die Bilder waren eine große Hilfe, um die Thematik zu verstehen, da sie sehr detailreich sind.“ (Übersetzung E. W.-J.) Ein anderer schreibt auf Deutsch: „Ja, ich fand die Bilder sehr nützlich. Sie machten den Text lebendig. “ Ein Student antwortet auf Norwegisch:

Dass wir auf eine Art eine Beziehung zu Lisa bekommen, führt dazu, dass wir mehr aus dem Buch lernen können. Wenn wir den Charakter kennen und verstehen, was der Charakter fühlt, dann ist es meiner Meinung nach einfacher, den Inhalt des Buches zu lernen. (Übersetzung E. W.-J.)

Dadurch, dass die Lerner sowohl Bilder als auch Texte verwenden können, haben sie die Möglichkeit mit Multimedialität zu experimentieren und die Intensität der Kommunikation zu steigern. Sie können den Einfluss weltgeschichtlicher Ereignisse auf eine relativ ,einfache“ Frau mitgestalten und dabei kommt auch Empathie ins Spiel, die über die literarische Empathiefähigkeit (Olsen 2011) hinausgehen kann. Ein Student drückt das auf dem 
Fragebogen so aus: ,„[...] Wir haben versucht, ein lebendiges Gespräch zu produzieren und es mit Einfühlung zu präsentieren, und wir haben davon gelernt. U. a., dass wenn du vor deiner Klasse etwas mit Einfühlung zu präsentierst, ist es einfacher, ihre Aufmerksamkeit zu bekommen."

Die Lerner haben sich demnach nicht nur in Lisa hineinversetzt, um ein lebendiges Gespräch zu gestalten, sondern das, was sie auf literarischem Feld erprobt haben, auch auf ihre Situation und ihre Kommunikation untereinander übertragen. Somit zeigen die Lerner, was die Auseinandersetzung mit Geschichte ,von unten ' anhand einer , einfachen' Frau für die Entwicklung von Empathiefähigkeit, Verständnis und Toleranz über das Buch hinaus leisten kann. 


\section{Nordic Journal of Modern Language Methodology}

$2018,6(2), 3-27$

\section{Bibliographie}

Afdal, Geir. Tolerance and curriculum. Conceptions of tolerance in the multicultural unitary Norvegian compulsory school. Münster, New York, München, Berlin: Waxmann, 2006.

Brandstätter, Ursula. Grundfragen der Ästhetik. Bild-Musik-Sprache-Körper. Köln: Böhlau UTB, 2008.

Brecht, Bertolt. Kalendergeschichten. Hamburg: Rowohlt, 2010.

Bredella, Lothar. „Wozu lesen wir Geschichten im Fremdsprachenunterricht? Zur Entwicklung von Empathie-, Kooperations- und Urteilsfähigkeit“. Zeitschrift für Fremdsprachenforschung, 2012, S. 3-31.

Breithaupt, Fritz. Kulturen der Empathie. Frankfurt a. M.: Suhrkamp, 2009.

Creswell, John W. Qualitative Inquiry Research Design. Choosing Among Five Approaches. Thousand Oaks: Sage, 2007.

Dammann-Thedens, Katrin und Magdalena Michalak. „Bildnarrationen im Fremdsprachenunterricht- Annäherungen an das Bildverstehen“. Zeitschrift für Interkulturellen Fremdsprachenunterricht 17:2, 2012, S. 129-142.

Ehlers, Swantje. Literaturdidaktik eine Einführung. Stuttgart: Reclam, 2016.

Geertz, Clifford. Dichte Beschreibung. Beiträge zum Verstehen kultureller Systeme. Frankfurt a. M.: Suhrkamp, 1987.

Hoel, Torlaug Løkensgard. „Forsking i eige klasserom“. Norsk pedagogisk tidsskrift 6, 1997, S. 379-387.

Kordon, Klaus und Peter Schimmel. Die Lisa Eine deutsche Geschichte. Weinheim: Beltz, 2002/2007.

Martinez, Matias und Michael Scheffel. Einführung in die Erzähltheorie. München: C.H. Beck, 2009.

Meisenberg, Norbert. Arbeitsheft. Die Geschichte des 20. Jahrhunderts, dargestellt an der Lebensgeschichte der Lisa. Weinheim: Beltz, 2004.

Olsen, Ralph. „Das Phänomen Empathie beim Lesen literarischer Texte. Eine didaktischkompetenzorientierte Annäherung“'. zaeb.net. 2011. http://zaeb.net/index.php/zaeb/issue/view/14 (Zugriff am 28.11.2017).

Postholm, May Britt und Torill Moen. Forsknings- og utviklingsarbeid i skolen. Metodebok for leerere, studenter og forskere. Oslo: Universitetsforlaget, 2009. 


\section{Nordic Journal of Modern Language Methodology}

Riemer, Claudia. „Empirische Unterrichtsforschung und Action Research“. In: Wolfgang Hallet und Frank G. Königs (Hrsg.), Handbuch Fremdsprachendidaktik. SeelzeVelber: Klett/Kallmeyer, 2010, S. 359-363.

Seel, Martin. Aktive Passivität. Über den Spielraum des Denkens, Handelns und anderer Künste. Frankfurt a. M.: S. Fischer Wissenschaft, 2014.

Spinner, Kaspar H. „Handlungs- und produktionsorientierter Umgang mit Kinder- und Jugendliteratur“. In: Günter Lange (Hrsg.), Taschenbuch der Kinder- und Jugendliteratur Band 2 Medien Ausgewählte Aspekte (4. Aufl.). Hohengehren: Schneider, 2005, S. 978-990.

Spinner, Kasper H. Kreativer Deutschunterricht, Identität, Imagination, Kognition (3. Aufl.). Seelze-Velber: Kallmeyer/Klett, 2008.

Thiele, Jens. Das Bilderbuch: Ästhetik, Theorie, Analyse, Didaktik, Rezeption. Oldenburg: Isensee, 2003.

Turner, Victor. The anthropology of Performance. Frankfurt a. M.: Fischer, 1988.

Weidenmann, Bernd. Psychische Prozesse beim Verstehen von Bildern. Bern, Stuttgart, Toronto: Verlag Hans Hueber, 1988.

Zimmermann, Holger. Geschichte(n) erzählen. Geschichtliche Kinder- und Jugendliteratur und ihre Didaktik. Frankfurt a. M.: Peter Lang, 2004. 


\section{Anhang}

\section{Anlage 1 Arbeitsaufgaben für die Sekundarstufe}

Die Lisa. Eine deutsche Geschichte. Klaus Kordon und Peter Schimmel (2002)

Aufgabe 1: Gruppe bilden, Gruppenleiter wählen, eine Lebensphase von Lisa wählen. Der Zeitrahmen, von dem das Buch handelt, lässt sich in sechs Abschnitte einteilen. Ihr könnt zwischen folgenden Gruppen wählen:

Oppgave 1: Danne gruppe, velge gruppeleder, velge en fase av Lisas liv. Tidsrammen boka handler om kan deles inn i 6 avsnitt. Dere kan velge mellom følgende grupper:

Gruppe 1: Die Kaiserzeit bis zum Ende des 1. Weltkrieges/Revolution. (1899-1919) S. 3-9

Gruppe 2: Die Weimarer Republik (1919-1933) S.10-13

Gruppe 3: Die Nazidiktatur (1933-1945) S. 14-19

Gruppe 4: Vom Kriegsende bis zum Wiederaufbau (1946-1955) S. 20-25

Gruppe 5: Das geteilte Deutschland. Bundesrepublik Deutschland und DDR (1958-1967) S. 26-31

Gruppe 6: Deutschland bis zur Wende (1970-1990) S. 26-31

\section{Die Lisa. Eine deutsche Geschichte. Klaus Kordon und Peter Schimmel (2002)}

Gruppe Nr:

Thema:

Navn på gruppemedlemmer:

Aufgabe 2: Wählt auf jeder Seite bis zu 5 Schlüsselwörter und 2 Ausdrücke aus, die euch gefallen. Oppgave 2: Velg på hver side opp til 5 nøkkelord og 2 uttrykk som dere liker.

\begin{tabular}{|l|l|}
\hline Seite: & \\
Unsere Schlüsselwörter auf Deutsch & Norwegisch \\
\hline 1 & \\
\hline 2 & \\
\hline 3 & \\
\hline 4 & \\
\hline 5 & \\
\hline Ausdrücke & \\
\hline 1 & \\
\hline
\end{tabular}




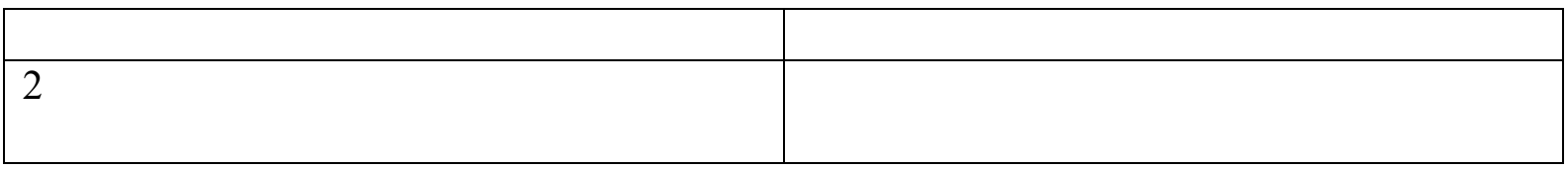

Aufgabe 3: Diese Aufgabe besteht aus drei Teilen. Denne oppgaven består av tre deler.

Bildbeschreibung: Wählt ein oder zwei Bilder aus eurer Lebensphase von Lisa aus und beantwortet auch die Fragen im Arbeitsheft zu eurem Bild/euren Bildern

Billedbeskrivelse: Velg en eller to bilder fra den tidsfasen i Lisas liv som dere har valgt og besvar spørsmålene som hører til deres bilde/deres bilder.

Tagebucheintrag: Wählt eine Episode aus eurer Lebensphase von Lisa aus und schreibt eine Notiz ins Tagebuch.

Dagboknotat: Velg en episode fra den tidsfasen i Lisas liv som dere har valgt og skriv et notat i dagboka.

Kreative Aufgabe: Wählt eine passende Überschrift zu eurer Lebensphase von Lisa.

A) Rollenspiel: Schreibt einen Dialog zu einer Episode aus Lisas Leben. Spielt den Dialog der Klasse vor.

B) Gestaltet eine Powerpointpräsentation oder einen kurzen Film, etc. zu eurer Episode aus Lisas Leben.

Kreativ oppgave: Velg en passende overskrift for den tidsfasen i Lisas liv som dere har valgt.

A) Rollespill: Skriv en dialog til en episode i Lisas liv. Spill dialogen for klassen.

B) Lag en powerpointpresentasjon, en kort film, fotostory, etc. om Lisas liv. 


\section{Nordic Journal of Modern Language Methodology}

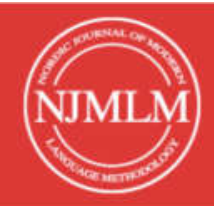

$2018,6(2), 3-27$

\section{Anlage 2: Vollständige Bildbeschreibung einer Lernergruppe aus dem \\ Sekundarbereich (Doppelseite 6-7 Kaiserzeit 1. Weltkrieg 1914)}

\section{Bildbeschreibung Die Lisa}

Wir haben das Bild auf Seite 6 und 7 gewählt. Das Jahr ist 1914 und der Krieg hat genau begonnen. Im Mittelpunkt sehen Sie die Lisa, und sie steht dort mit ihrem Vater und ihrer Mutter. Es scheint, als ob sie einander sehr lieben. Wenn ein Krieg beginnt, erwartet man instinktiv traurige Gesichter und herzzerreißende Abschiede. Ganz im Gegenteil, gibt das Bild ein Eindruck von positive Gefühlen. Die Menschen lächeln und winken den Soldaten, die in den Krieg ziehen, mit ihren Hüten. Der Vater von Lisa scheint auch nicht traurig zu sein. Das macht das Bild ein bisschen sonderbar.

Auf den ersten Blick, sind es wahrscheinlich die Fahnen, die der Aufmerksamkeit von dem Leser erregen. Die Fahnen sind schwarz, weiß und rot. Diese Fahne war die Flagge des Norddeutschen Bundes. Die Farben sind im Kaiserreich die akzeptierten Nationalfarben der Deutschen. Es gibt verschiedene Theorien von dem Ursprung der Flagge. Die Erklärung, die die meisten glauben, ist dass die Schwarz-Weiß-Rot Fahne hat schwarz-weiß von der schwarz-weißen Flagge der Preußen genommen, und weiß-rot von der traditionellen weißen und roten Flagge der Hansastädte.

Die dominierende Farbe ist gelb, und das ist nicht nur auf dieser Bild. Auf allen Bildern während der Krieg gibt es immer gelben Himmel (Seite 6-9). Wir wissen nicht, warum aber wir haben in Norwegisch gelernt, dass gelb die Farbe der Fälschung ist. Das kann Sinn machen, weil es ganz falsch ist, was der Kaiser gesagt hatte. Der Krieg ging nicht zu Ende an Weinachten, und Deutschland konnte den Krieg nicht gewinnen. Ob die gelbe Farbe diesen Effekt haben soll, wissen wir nicht, aber es ist eine mögliche Erklärung.

Auf der rechten Seite des Bildes gibt es einen Mann, der auf eine Leiter geklettert ist. Er hat ein Schild, was „Feldherrenweg“ heißt. Ein Feldherr, auch Heerführer genannt, ist der höchste Militär im Krieg. Es ist kein Zufall, dass dieses Schild während des Krieges an der Hauswand angebracht wird. Dieser Straßenname wird auch mehrmals später in dem Buch aufgeführt.

Das war ein kurzer Text über unsere Gedanken von dem Bild auf Seite 6-7. Das wichtigste ist der Krieg, der gerade begonnen hat, und wie die Leute auf das reagieren. Man erwartet Tränen aber das Gegenteil war die Realität. 


\section{Anlage 3: Fragebogen für den Sekundarbereich}

Die Lisa. Eine deutsche Geschichte. Klaus Kordon, Peter Schimmel (2002)

Besvar spørsmålene så utførlig som mulig. Du kan svare på tysk eller norsk. Beantworte die Fragen so ausführlich wie möglich. Du kannst sowohl auf Deutsch als auf Norwegisch antworten.

1) Welchen Zeitabschnitt und welche Seiten im Buch hast du/deine Gruppe bearbeitet?

Hvilket tidsavsnitt og hvilke sider i boka har du/din gruppe arbeidet med?

2) Warum hast du/ habt ihr diese Seiten im Buch gewählt?

Hvorfor har du/ dere valgt disse sider i boka?

3) Haben die Bilder im Buch dazu beigetragen, dass du die Thematik von deinem Zeitabschnitt besser verstehen konntest? Wenn ja, wie?

Har bokas bilder bidradd til at du forstod tematikken i ditt tidsavsnitt bedre? Hvis ja, hvordan?

4) Kannst du die Protagonistin (Hauptfigur) etwas näher beschreiben? Beschreibe u. a. was für eine Persönlichkeit sie hat. Wie zeigt sich ihre Persönlichkeit? Kannst du ihre Handlungen und ihre Reaktionsmuster verstehen? Diese Fragen beziehen sich auf das ganze Buch!

Kan du beskrive protagonisten (hovedfiguren) litt nærmere? Hva slags personlighet har hun? Hvordan viser hennes personlighet seg? Kan du forstå hennes handlinger og reaksjonsmønstre? Spørsmålene gjelder hele boka!

5)In deiner Arbeit/eurer Arbeit musstest du /ihr entscheiden, wie ihr die Hauptfigur darstellen wolltet. Was war für dich/euch wichtig?

I ditt/deres arbeid måtte du/dere ta en avgjørelse hvordan dere vil framstille hovedfiguren. Hva var viktig for $\mathrm{deg} / \mathrm{dere}$ ?

6) Wie hat dir/euch die Arbeit mit einem Bilderbuch gefallen?

Likte du/dere å arbeide med en billedbok?

7) Was hast du durch deinen Beitrag/ durch deine Aktivität/ anhand deiner Untersuchungen über das Thema gelernt? Schreibe kurz, was du gemacht hast, und was du dadurch gelernt hast.

8) Hva har du lært av ditt bidrag/ din aktivitet/ din utforskning av temaet? Skriv kort, hva du har gjort og hva du har lært gjennom det.

9) Was hast du durch die Präsentationen von deinen Mitschülerinnen und Mitschülern gelernt?

Hva har du lært gjennom presentasjonene av dine medelever? 


\section{Nordic Journal of Modern Language Methodology}

10) Meine weiteren Ansichten und Bewertungen zur Arbeit mit dem Bilderbuch Die Lisa. Hier kannst du ergänzen, was du wichtig findest, und was die anderen Fragen nicht beantwortet haben. Mine synspunkter og vurderingen av arbeidet med billedboka Die Lisa. Her kan du tilføye, hva du synes er viktig og hva de andre spørsmål ikke har gitt svar på. 